\title{
Lessons We Learned Designing and Building the Chandra Telescope
}

\author{
Jonathan Arenberg (1) and Gary Matthews (2) \\ \&
}

C. Atkinson (1), L.Cohen (4), C. Golisano, K. Havey, K. Hefner (3) ,C. Jones (4), J. Kegley (3) , P. Knollenberg (1), T. Lavoie (3), J. Oliver (3), P. Plucinsky (4) , H. Tananbaun (4), S. Texter (1) and M. Weisskopf (3)

(1) Northrop Grumman Aerospace Systems, One Space Park, Redondo Beach CA 90278, USA

(2) Excelis, 1447 St. Paul Street, Rochester NY, 14606-0488, USA

(3) NASA Marshall Space Flight Center, Huntsville AL, 35816 USA

(4) Smithsonian Astrophysical Observatory- Center for Astrophysics, 60 Garden Street, Cambridge MA USA

\begin{abstract}
Chandra X-ray Observatory. This paper offers some of the major lessons learned by some of the key members of the Chandra Telescope team. We offer some of the lessons gleaned from our experiences developing, designing, building and testing the telescope and its subsystems, with 15 years of hindsight. Among the topics to be discussed are the early developmental tests, known as VETA-I and VETA-II, requirements derivation, the impact of late requirements and reflection on the conservatism in the design process.
\end{abstract}

Key Words: AXAF, Chandra, $x$-ray telescope, space missions, lessons learned

\section{Introduction}

This paper is work of many contributors and constitutes a survey of lessons learned from many of the principals involved in the management, design, build and test of the Chandra telescope. The scope of these lessons is in the engineering, testing and implementation of the telescope. Other authors in this conference will offer views of the science and operations lessons learned and other examples can be found in the literature. [1,2] Our engineering lessons are delivered from the vantage point of retrospect of nearly 15 years. It is our hope that the filter of time has helped the really important lessons to be separated from the more trivial. The process for collecting the lessons in this paper was simply to "put out the word" through the network of AXAF/Chandra alumni then collect and edit the responses. We make no pretense that this set of lessons is has been gleaned from every discipline and organization involved, but an attempt was made to be as comprehensive as possible.

This paper is organized in various categories of lessons, not chronologically, or by contributor. This way our lessons come to you, the reader, from the Chandra team, no individual contributors

We give a very brief historical overview of the program, and introduce the components of the Chandra X-ray Telescope to place our comments and lessons in proper context. 


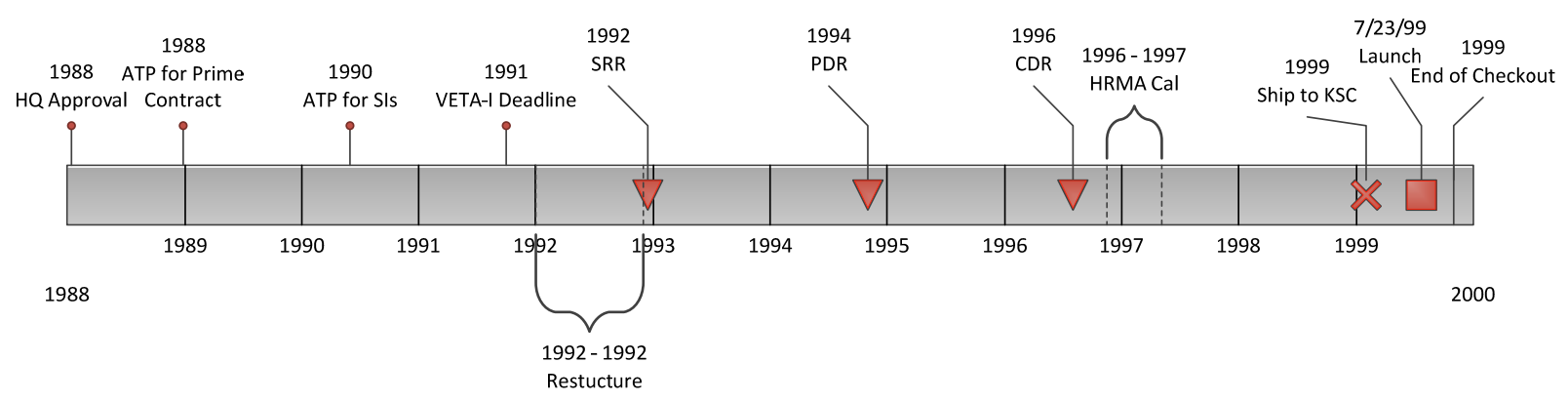

Figure 1: Top Level AXAF/Chandra Program Timeline

A key event in the program, both technologically and in terms of forging a true team was the VETA-I. As a result of agreements made between NASA and the US Congress, the x-ray performance of the largest pair of mirrors, $\mathrm{P} 1$ and $\mathrm{H} 1$, had to be demonstrated, to the 0.5 arcsec full-width half maximum level by the deadline of the end of fiscal 1991. [3] Failing to meet this deadline meant program cancellation. VETA-I was supremely challenging, as the team needed to manufacture the mirrors, mount them, get a new test facility online and into this new facility integrate a new set of $x$-ray detectors and alignment metrology. [4,5] The entire program was focused on the VETA goal and was supported by NASA program and Center management. The entire VETA-I experience was in the words of Arthur Wellesley, First Duke of Wellington, "It has been a damned serious business.... It has been a damned nice thing - the nearest run thing you ever saw in your life." Just as the Battle of Waterloo made the Duke of Wellington and post-Napoleonic Britian, the VETA-I test made the program team, from that day to the present. We coalesced from dedicated engineers, scientists and managers into the AXAF (Chandra) team.

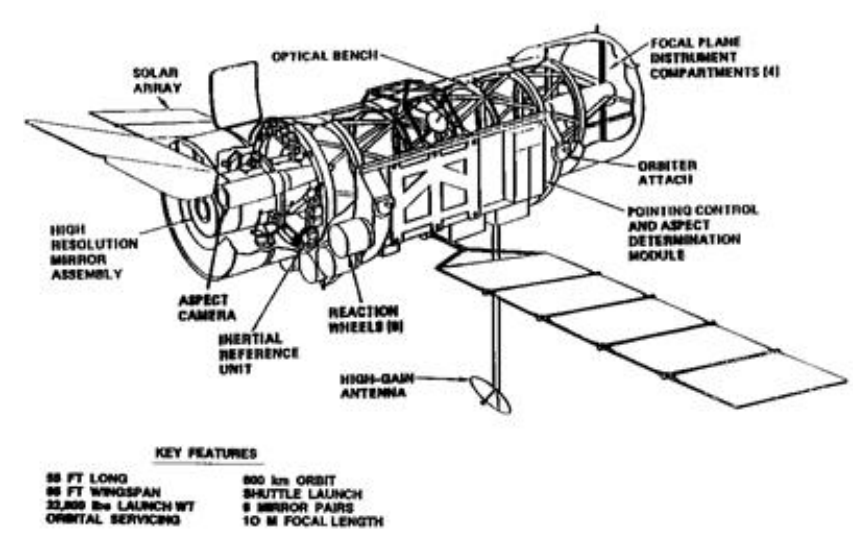

Original Mission Concept

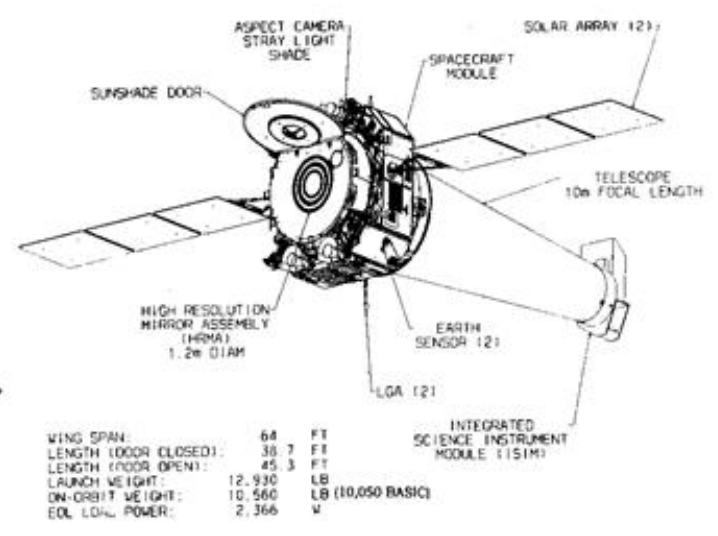

Flight Mission Concept

Figure 2: Original and Reformulated Flight Concepts

Despite the triumph of the VETA-I test, another challenge lay before the team, restructuring. Shortly after the VETA-I test, managers at NASA HQ determined that the life-cycle costs for the baseline serviceable program was too high. The resulting study to reformulate, or restructure the program took the attention and work of the entire team. The resulting mission is essentially what the system that was launched, whose flight anniversary this paper celebrates. The original mission called for a $32,000 \mathrm{lb}$ space craft, that was in a man-serviceable space shuttle, low earth orbit, with an articulated HRMA over an array of replaceable focal plane instruments. The final configuration is a $10,500 \mathrm{lb}$ spacecraft, that is in an eccentric orbit, with a fixed complement of instruments, and is not serviceable. [4] More of the details of this effort can be found in the literature. [5] 


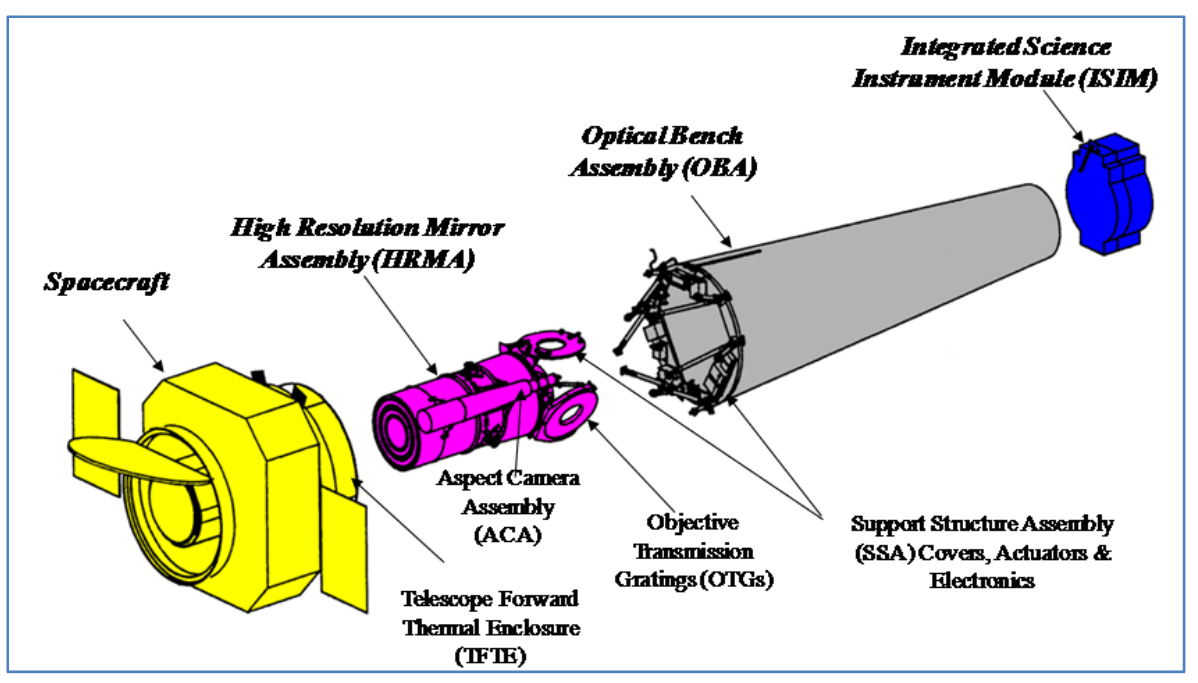

Figure 3: Major Subsystems of the Chandra X-ray Observatory

\section{Lessons Learned}

\section{Team}

From all of the respondents, the single most important aspect of our ultimate success was the development of a truly integrated team. This team is made of every skill

areas needed for the mission. This team was about as "badge-less" as humanly possible, with industry, government, and university communities working together with a common goal - the ultimate success of the mission. Words that come to mind to characterize the team effort include: respect, cooperation, collaboration, dedication, perseverance, pursuit of excellence with recognition that perfection was neither an achievable nor a reasonable goal, and relevance of budget and schedule along with performance and specifications.

Out team did not start out this way, but after many face to face meetings, tests and shared challenges it did emerge and remains to this day. The spirit of this team is evidenced years after launch in this paper.

\section{Requirements}

A key ingredient in Chandra's overwhelming scientific and programmatic success is the fact that Chandra was a science-driven mission whose scientific requirements barely (if at all) changed throughout the time of its pre-development, i.e. from 1977 to 1992.

When the science Chandra should do was determined, the means to build the optics and principal detector system (X-ray sensitive CCDs). The mission requirements were based on what was needed to accomplish the science, not what could be done. We did not adhere to the "I'll build it, you will use it" philosophy of so many NASA and indeed International missions. There were some practical limitations, the Shuttle envelope guide/limit the design but, it did not limit the science. ${ }^{1}$ The $1000 \mathrm{~cm}^{* *} 2$ and the subarcsecond resolution necessary to resolve the background were not influenced or impacted by the Shuttle envelope.

Furthermore, we as a community acted responsibly and with vigor to avoid "creeping requirements". The only major requirement that was changed through the birth of the mission through launch was the deletions of the man-servicing requirement. This major requirement change wasn't an example of requirements creep but requirements drop thanks to the multi-billion dollars of projected servicing costs that were saved. In order to prevent "creep" all stakeholders were involved in any discussions involving requirements. For example, potential development and operations contractors, other involved NASA Centers, scientific/user institutions, MSFC technical organizations and NASA Headquarters.

There are two areas where requirements needed to be changed or added. Telescope alignment and shielding or background suppression. Shortly after reformulation and leading into PDR, the first specification for the ISIM [5] was written. At this point in the program, it was clear that complete

\footnotetext{
${ }^{1}$ The Chandra payload was the longest item launched from the bay of a space shuttle in the history of the shuttle
} program. 
reassessment of the telescope alignment requirements was necessary. A comprehensive effort was undertaken to derive the proper alignment requirements for the restructured program. This revision, which took approximately 18 months to complete, involved all stakeholders, and resulted in an affordable, executable and well performing telescope. The team did not do so well in considering requirements for background suppression, including stray light, high energy cosmic rays and charged particles focused by the mirrors. All of these requirements were for some reason implemented late in the program. The stray light requirement turned out not to be an issue, since the HRMA thermal baffle which is designed to keep heat in also solves the dual problem of keeping stray radiation (heat or light) out of the HRMA. The requirement to shield from high-energy particles coming through the bench, and charged particles focused by the HRMA, came at the point in the program when the HRMA, ISIM and OBA were built. Tantalum baffles and a magnetic broom were implemented to mitigate the background but due to the late introduction of the requirement, not all background sources could be mitigated, especially protons. (See the radiation section below for the consequences.)

\section{Systems Engineering}

Spend the resources to provide for a strong system engineering organization throughout the program. Assign managers for all interface control documents and layout a schedule for their development through baselining. We held regular face to face interface meetings. A key outcome of the technical interchange meetings was the development of strong Interface Control Documentation. The documentation laid the ground work and maintained a well-defined road for the design teams in the development of the facilities, test and special equipment for Chandra. The resultant documentation spanned across many organizations and as a result fostered strong team work and coordinated efforts from all of the companies, SAO and MSFC

Make sure the systems engineering manager has direct control of all manpower for systems analyses such as thermal, structural dynamics, error budgets, loads, mass properties, electrical power demand, orbital mechanics, etc. Do not overlook the need for strong systems engineering involvement in requirements and verification traceability.

Make sure that one organization is responsible for end-to-end systems engineering for the entire payload including the payload ground operations system. Engineering of the entire system includes penetrating the total system design on both sides of and ICD, not just working up to your side of it. When responsible for building a total systems analytical model (thermal, Structural, Dynamic, Electrical, etc.) by assembling a number of analytical inputs from other organizations, systematically check the accuracy of these inputs before using them and perform sanity tests on the total model with simple yet carefully chosen boundary conditions to make sure it is correct.

When verifying performance of optical elements or systems always cross check the results using another completely different test method. When required to use gravity off-loaders in the test of optical systems, it is essential that they be rigorously tested and correlated with analytical

models under both nominal and off nominal conditions.

\section{Cross Checks}

Cross-checks are a great tool that can be implemented very simply

Chandra came on the heels of the Hubble Space Telescope, so the sensitivity to having an error in the telescope was extremely high. Opportunities presented themselves to implement some metrology 
crosschecks during the assembly operations as a means of having an independent look at a particular parameter using a completely different measurement method than the primary verification approach. Two simple examples were the focus cross check and the mirror deformation cross check.

For the focus cross check, the focus of the HRMA was established relative to an Optical/Mechanical reference located at the node of the telescope. A Shack-Hartmann sensor located at the focal surface was used to align the optics to the Optical/Mechanical reference. During the X-ray Calibration measurements of the HRMA, sensors were located at the focal surface relative to a second Optical/Mechanical reference. Finally, the focal surface of the integrated telescope was established using the same ShackHartmann sensor and Optical/Mechanical reference to which the mirrors were originally aligned. A simple cross check was envisioned to accurately measure the distance between the X-ray Calibration Optical/Mechanical reference and the focal surface used during that testing with a laser distance meter. Then, during Telescope integration, after the focal surface had been set, the X-ray Calibration Optical/Mechanical reference was swapped in, and the laser distance meter was used to measure the location of the focal surface relative to it. A simple check that took a day and yet built significant confidence that the focal location was set correctly.

The mirror deformation cross check was also very simple. Supporting the mirrors during installation was, by necessity, very precise in order to preclude deforming the mirrors. The most likely deformation shape of the mirrors was an ovalization of them, or a form of ovalization where the cylinder is an oval at one end, and an oval at an orthogonal orientation at the other end. The way this was controlled was by very precise load cells and precision bearings to ensure no unwanted loads were introduced into the mirror. A simple cross check was implemented using the Shack-Hartmann sensor. It took 24 samples around the aperture of the mirrors in order to establish the focus, coma, and astigmatism terms to aid in alignment. By simply looking at more of the coefficients from the Fourier transform of the 24 samples, ovalization of the mirrors could readily be detected. This provided a very simple crosscheck that the mirrors had not been deformed during the installation process.

These kinds of crosschecks can be implemented readily as a means to boost confidence in the performance and/or to catch workmanship errors that might otherwise go undetected.

Any mission critical verification that is verified in whole or in part by analysis should be double checked or preferably analyzed in parallel by an independent party. This same principle should also apply to any operational commands that are generated based on an analytical input.

To the extent that resources permit, design the system with reserve capability, including failure tolerance, beyond that stated in the requirements.

Never stop looking for undetected failure modes or potential problems throughout the entire program development and operational phase. As soon as you start feeling comfortable Mr. Murphy will call.

Be especially cautious when dealing with highly complex scientific hardware where the developer is "the only one that understands how it works". This system should not be exempt from review by as knowledgeable a team of experts as you can assemble, even if they have to be brought in from another NASA center or independent organization. (Remember the ACIS CCD problem. The shortcomings of the radiation testing may have been caught by such a team, if we had of assembled one early in the program.)

\section{Design Process}

Early analysis of telescope design architectures, even with a small group of people, saves time (not going down a path that will not lead to a good design) when you get in Phase B. Even those those paths may not 
have proven successful, the knowledge gained by the engineers becomes invaluable. These early design studies found many many ways to realize a poor design and this knowledge ultimately lead to a clearly excellent design.

When designing a complex, high performance systems, such as Chandra, no effectshould ever be written off as not being important until you have done at least a simple calculation or ] Leon Van Speybroeck, telescope scientist put it, a Gedankenexperiment. The analyst must make sure the simple calculation or thought experiment have the correct and important parameters. The lesson here is deal with issues in a quantifiable, not an emotional or political manner.

New technology (graphite composites for example) can lead to more problems than it can solve. Old time materials and processes that have worked in the past can still work. The High Resolution Mirror Assembly utilized a periscope for fine guidance; transferring the pointing of the center part of the HRMA to the aspect camera (AC). This periscope had to maintain its shape over small temperature excursions. The fold mirrors that were part of the periscope had to be supported by the composite tube metering the distance and angle between the HRMA and AC. One might think that the mirror support to composite tube I/F should also be composite. While an all composite design showed it met its performance requirements, there was a small part whose design and manufacture had to be quite precise (and would have been challenging to make). It was shown that if this I/F was changed to simple Invar, the performance improved by almost 10X.

The designer must consider the assembly schemes that will be used and properly account for assembly stresses. Some composite parts are Chandra were manufactured in 120 degree circumferential segments that needed to be spliced together to form a full 360 degree functionally stiff structural component. The parts were assembled with 3 doublers but the initial shape of these "flimsy" doublers caused them to have to be forced into the correct shape during assembly. A later failure of one of these joints was found to be caused by poor understanding of the assembly processes and tolerance needs.

When you have to manufacture a high quality optic and you think you are finished with its manufacturing but have to cut it to length (from is uncut length of $\sim 1.1 \mathrm{~m}$ to its final required length of $\sim 1.0 \mathrm{~m}$ ) you have to prove out far in advance what are the expected residual stresses in the glass (that would cause the figure of the optic to change after it is cut to length). While manufacturers may suggest that their glass is stress free, that statement has to be taken with a grain of salt. $0.07 \mathrm{MPa}(10 \mathrm{psi})$ residual stress may be perfectly acceptable for stiff optics but not For optics what have some geometric ratio of 40 to 1 . Not only does the residual stress in the bulk material affect the outcome but so does the way the cutting operation and post cut finishing operations are performed. Not considering these effects caused us to have to go back and repolish more than one of the 8 flight optics after this process was completed.

The HRMA mirrors are supported by up to $12,25 \mathrm{~mm}$ to $40 \mathrm{~mm}$ diameter invar pads that are epoxied to the outside diameter of the mirror. Analysis of the epoxy showed that over seasonal humidity cycles the strain in the adhesive markedly changed. This knowledge lead to the requirement to control humidity to be dry during the bonding operation. So over the months and years that the HRMA lived on earth before launch, its figure might change, but when in orbit and dried out, the figure would return close to its assembled condition. A method also had to be found to remove excess water from the epoxy's resin component, a molecular sieve was identified, that would absorb water and only water from the neat epoxy resin. Optical measurements of the HRMA during its X-ray testing at NASA/MSFC X-ray facility (hard vacuum, room temperature test) seemed to confirm our analysis and implementations. Moral of the story is make sure you understand how all material properties can affect you, no matter how small.

While analysis is important and indeed critical in the early stages of a program, excessive focus on analysis later in the program can lead to "analysis paralysis". This particular affliction was avoided by careful management. This does not mean that management was blind to new analytic problems. Well into the 
design of the spacecraft and after completion of the HRMA, careful analysis revealed that the reaction wheel disturbances would have excited resonant modes in the HRMA and ruined imaging performance. Damped isolators, which were not originally planned in the program were implemented and solved the issue.

\section{Risk Retirement}

In any development effort, especially when new technologies, materials or applications are involvoed, Development Units, Engineering Units, and/or Qualification Units are produced to ensure the as built hardware behaves the way the design engineer envisioned it. This is a very valuable process; however, when building a new, complex system like Chandra, these models are necessary, but not always sufficient. It is very important to demonstrate integration, assembly, and test processes, procedures, and equipment before attempting them on the flight hardware. The Verification Engineering Test Articles (VETA), produced for Chandra, were key to the successful development of the Chandra Telescope.

During the early stages of the Chandra design development, it was realized that Kodak's traditional mirror mount design would not work for the configuration and design parameters of this x-ray telescope. Early funding (prior to official program start) was provided and a series of analyses and tests were conducted to identify a high quality bonding material and mount configuration that would satisfactorily support the optics while meeting their performance requirements. The program's decision to provide the funding based on the proposed study was key in jump starting the design efforts for the HRMA. Additionally because this design parameter was evaluated early on, it provided further opportunities to refine and qualify the concept with the test articles.

Kodak established an early contract with Hercules to develop and design the graphite epoxy sleeve concept that along with a new flexure and mount pad design would support the HRMA's mirrors at their neutral axis. This design provided very little distortion to the optics during both the assembly phase and their transition to an on-orbit environment. Many interactions, interfaces, and designs were bantered about between the companies that tied in each company's expertise to make the concept successful. Establishing the contract early and working the issues in a team oriented approach was critical to its success.

Due to the nature and size of the Chandra telescope as well as all of the coordination and "handshakes" that were required amongst the involved companies supporting this program, a well-defined and agreed on interface set was required and needed to be developed. TRW had the lead on the interface development

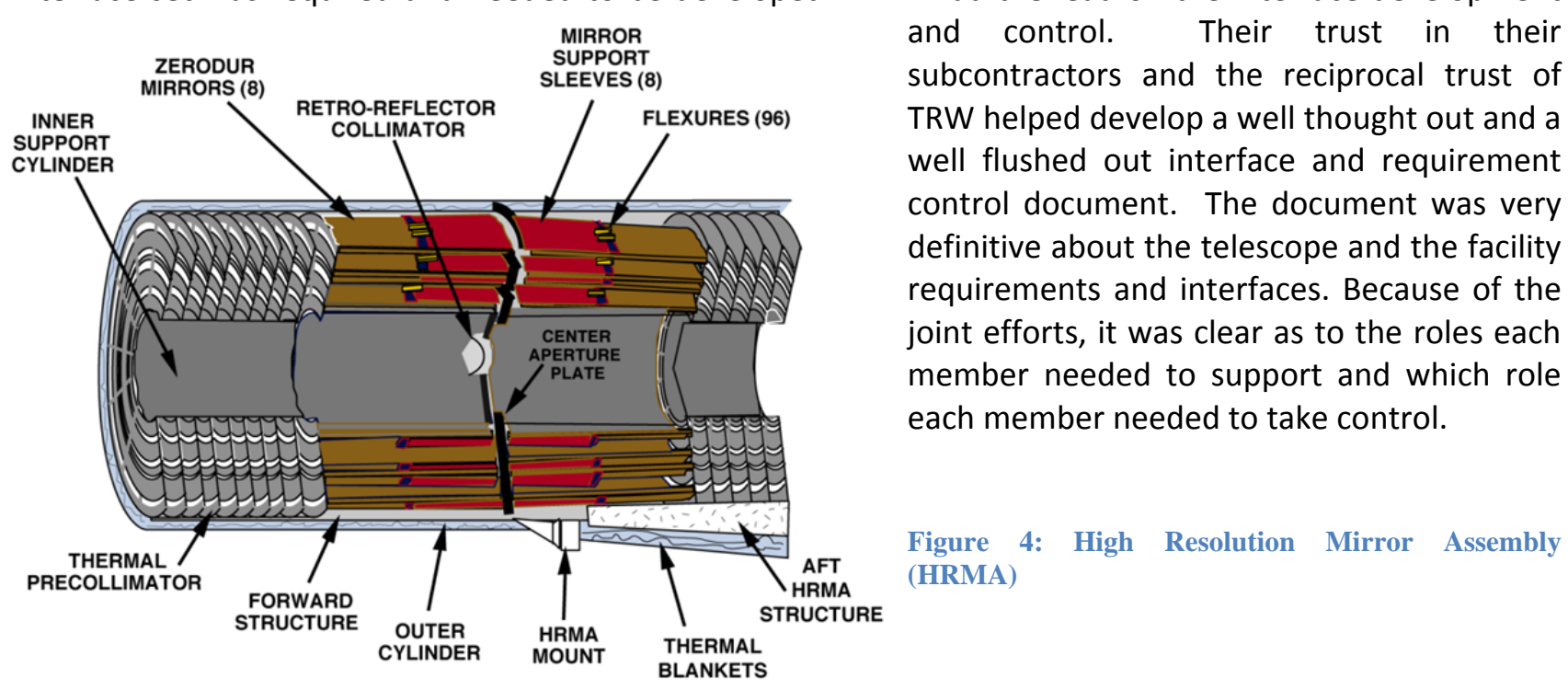


The optics in the High Resolution Mirror Assembly needed to be supported relatively strain free. That would provide the best success in meeting the extremely tight optical performance requirements. Because of the space and configuration of the optics, blade flexures were the design decision. As a result the machining of the titanium was critical to such thin bladed flexures. The machining had to provide the proper thickness tolerances but also provide a machined surface that would not allow propagation of any cracks that could result in a failure. Kodak was evaluating many different processes that involved wire electric discharge machining. The issue was the subsurface cracks, relative to the thin flexure blade, that left behind could cause a flexure failure under a launch load. Engineers at the SAO researched and found a small company that could conventionally machine flexures thus eliminating the subsurface micro cracks on the blades. Again, the teamwork amongst companies and organizations discovered and developed an approach that would contribute to the success of the program.

In addition to flexure development, a method to be able to remove an optic after it was bonded in place to those flexures was required. The reason; should some unforeseen event occur where the optic was either damaged or improperly supported, it needed to be removed efficiently. The bond pad was configured in a fashion to be mechanically removable from the flexure blade. Again, the tight space and the design configuration provided tight parameters creating a design challenge. The inherent issue with that was; a mechanical joint provided the potential of unwanted instability that added risk in meeting the extremely tight alignment requirements. Through a series of joint design reviews and technical interchange meetings between TRW, Kodak and SAO, as well as a series of analyses and tests, a design pulling together the results of all of those efforts was established that provided a concept that met all of its requirement, space constraints and minimized the potential impact to the optical alignment of any of the optics.

The HRMA is completely supported, both mechanically and optically, by its center aperture plate (CAP). The plate was to be the supporting backbone of the HRMA, provide controlling apertures for the optical path and provide a thermally stable environment for the optics. Research for several, high strength yet thermally conductive and machinable materials for the CAP was performed. The material also had to end up being extremely stable after processing because the entire optical alignment of the HRMA was grounded at the center aperture plate. After some finite element analysis, material trades and vendor research, a material selection was made that provided much of the attributes needed for this design. The next issue was to make the material stable after machining. One of the reasons the material was selected was its capability to be cryogenically stabilized. Kodak worked with a local company that had the capability to machine the material to the high tolerances. The local company also had connections to a facility that could provide the cryogenic stabilization at the temperatures required and at the size of the center aperture plate. Mock ups and trials were then conducted along with precise machining and measuring technique development. The combination yielded a machining and stabilization process that provided a center aperture plate that met all of its performance parameters.

Because the CAP was the critical alignment feature of the HRMA and because the HRMA was to perform in a zero gravity environment, techniques had to be developed to support the center aperture plate in a manner that best simulated its on-orbit condition. The optics were extremely heavy and although the CAP was more than suitable to perform all of its functions, it was not very stiff. That led to more deflection than allocated for the optical alignments. A support approach that included off-loading the weight of the optics during alignment and after securing them to the center aperture plate was needed. The challenge involved a low mass solution on the center aperture plate but it required hardness and repeatability. Two different design teams within Kodak needed to support this. The CAP design team and the off-loading mechanism design team. The key was to put as little equipment on the CAP as necessary yet provide the interfaces required to properly support the center aperture plate. The two teams worked together to develop and refine a design. The proof in the pudding was to perform the alignment approach on a full scale test article. That test article was critical to the techniques, manufacturing and test process development. It allowed a full trial run, a flush out of issues and a development of refinements for the actual build and alignment of the HRMA. 
The optics were supported on the center aperture plate but now that load needed to be carried somehow to the rest of the telescope. Additionally, the support for that load needed to carry the full launch and orbit insertion load of the HRMA into the telescope. Although this may seem to be a simple solution, the support needed to be attached to the center aperture plate with minimal residual hardware and not impart any external forces onto the center aperture plate that could potentially impact the optical alignment. The design was fabricated from a variety of design concepts used to support the optics on their flexures. The same bond material used to hold the optics was used to hold the main cylinder to the center aperture plate along with similar pinning techniques used between the mirror mount pads and the flexure blades. Also the close out cylinders that were secured to the main cylinder were bonded in place with a repeatable mechanical fastener release mechanism in the event a closeout cylinder needed to be removed. These design concepts were easily developed because of the early on work that was performed on the optic/flexure design development effort. Without those early on efforts, these concepts could have again been designed but the additional time an effort to development them in all likelihood would have caused some schedule delays.

The HRMA mount was the interface between the HRMA and the rest of the telescope. It provides the support of the HRMA before and after its optical alignment, during its transportation efforts within facilities and across facilities, and the HRMA's handling at a variety of times. Many of the pieces of support equipment were in their infancy of design as well as the final technique for the HRMA attachment to the remainder of the telescope. The mount design needed to support a variety of possible attachment techniques while meeting its own development schedule. It was decided that the mount design first and foremost needed to ensure loads would be carried out of the HRMA in the desired "neutral axis" position. By making that the fundamental requirement and assigning it configuration control, the remainder of the design was created to provide simple interfaces for all of the other configurations and no matter what configuration was employed the load always went through the neutral axis minimizing any loading conditions that could cause an optical misalignment.

\section{Verification Engineering Test Articles, VETA-I and VETA-II}

The VETA-I originally address the high risk early in the program, demonstration of the ability to manufacture the optics to the correct tolerances. The VETA-I also allowed for an early test of the test facility, ground support equipment and the core of the $x$-ray test team that stayed basically intact throughout the program.

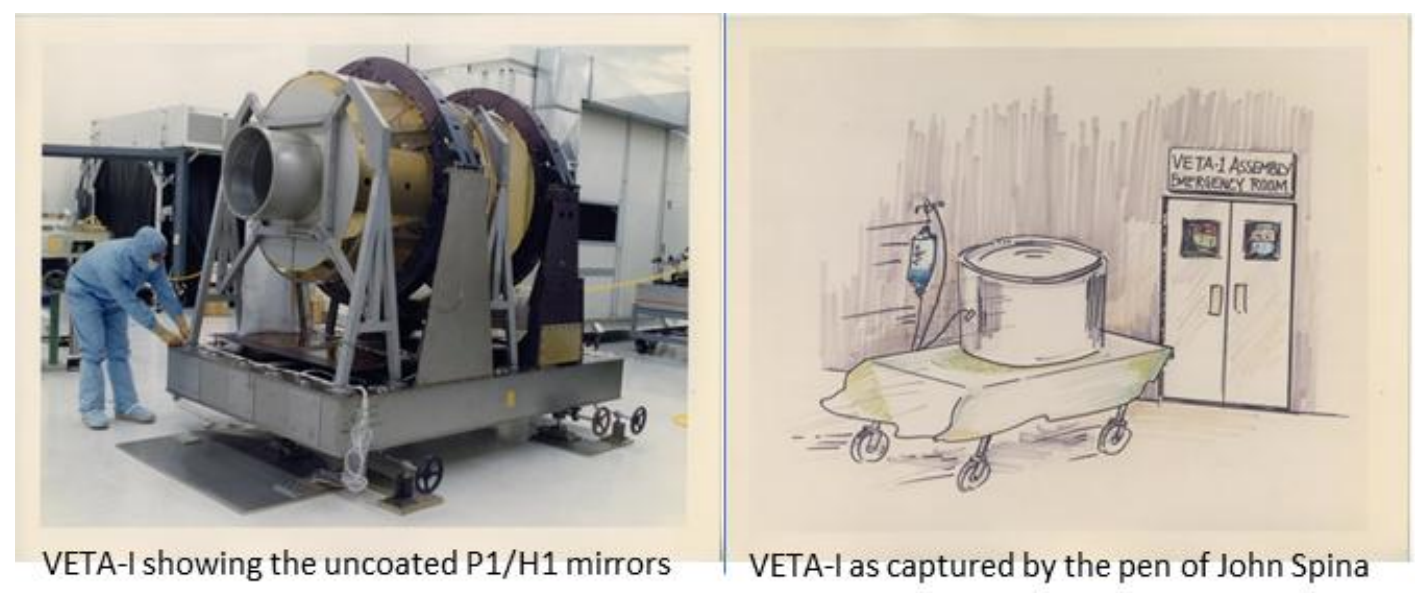

Figure 5: VETA-I Hardware and artistic interpretation 


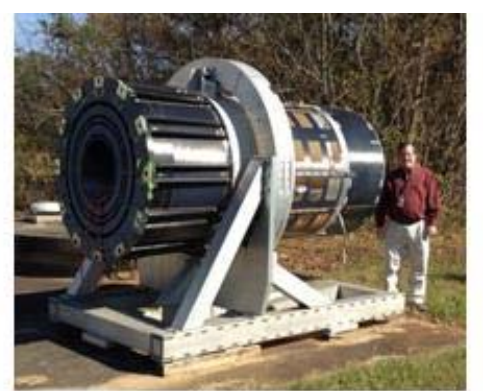

VETA-II as recently discovered at MSFC. Dr H. Jones, Jr is visible at right

Figure 6: VETA-II rediscovered in a warehouse at MSFC
VETA-II was a high fidelity simulator of the HRMA, the optical heart of the Observatory. The VETA-II effort took the largest two mirrors and aligned and mounted them to the flight tolerances using the flight handling hardware and alignment metrology. Hundreds of lessons learned were documented as a result of the VETA-II effort, resulting in changes to handling procedures, support equipment, metrology hardware, and alignment and metrology processes. This enabled the installation of the flight mirrors to go very smoothly, and resulted in the telescope being completed 1 month ahead of schedule. The VETA-

II is the very definition of early testing and good risk mitigation. It is interesting to note in hindsight that, VETA-II was always on the chopping block when budgets were tight, the mission would have

had a much lower probability of success without it. VETA-II gave us the design insight that our mirror bonding process was flawed due to a very subtle cure shrinkage that was not apparent until observed. Without this key insight, mirror alignments would have been impacted with severe schedule impact or a much lower performance optical system.

\section{Mechanisms}

The high and low energy objective transmission gratings (HETG and LETG) and the HRMA Aft Contamination Cover (ACC) are attached to the back of the HRMA. The mechanism development program benefited from several ground test activities.

While operating the HRMA contamination covers in a GSE mount at XRCF, we identified thermal tape inadvertently overlapped from the cover to the HRMA precollimator interface. The GSE mechanism was able to overcome the stiction from the tape, but the flight spring-loaded design most likely would not have sufficient torque margin to release the cover. This XRCF cover checkout step was not a formal test, but still identified a potential mission failure scenario. As a result, more extensive inspection of the flight hardware was incorporated when $1^{\text {st }}$ motion testing was completed at final assembly.

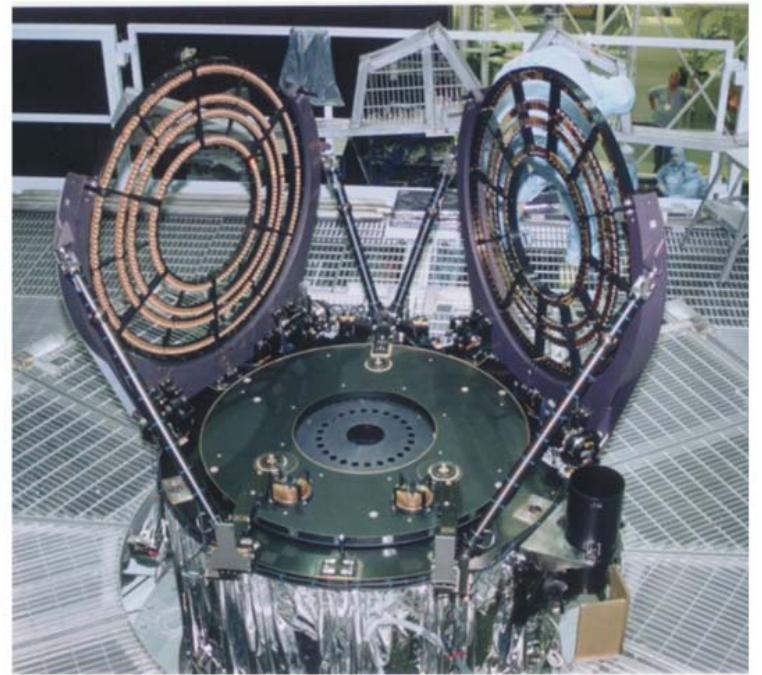

Figure 7: Grating and contamination mechanisms attached to the HRMA

Significant effort was spent in developing a robust mechanism ground test program with high fidelity flight cables, EM electronics, a flight computer simulator, and the mechanisms integrated as shown above. The primary and redundant mechanism and electronics features worked successfully, but while testing the mechanism failsafe feature, interference was discovered at the failsafe drive arm. This failure scenario could only occur within a limited range of motions that would be difficult to identify even with today's 3-D CAD tools. All deployment tests, even demonstrations of capability, can reduce program risk. Test at both subsystem and system levels of assembly are recommended.

\section{Thermal Control System}


Computer-based control was chosen early in the Chandra design to provide flexibility to address multiple operating modes and to provide customizable control to address degradation in electronics or thermal finishes over the course of the mission. Desirable features enabled with computer control included load management, setpoint and deadband adjustability, control interval options, and software-based crossstrapping.

The thermal software initialization process takes longer than normal operations as all control parameters and database values are loaded in memory. In the first ground TVAC test we discovered that the heaters were powered on at software startup, and the relatively long thermal software startup sequence allowed the high powered heater zones with low thermal mass to overheat. Although the heater zones were still below their design capability, in a warmer condition such as could occur on-orbit, this could have been a more serious issue if a thorough TVAC test had not identified the risk.

Since the heater setpoints are adjustable, the Chandra on-orbit operations team developed scenarios to help with peak voltage management by increasing optical bench heater setpoints prior to eclipse entry. Since the software included three addressable data bases, reconfiguration between modes has been straight forward. A flexible programmable thermal control system is a worthwhile investment for major missions like Chandra. The test program must exercise high fidelity simulations of on-orbit operational modes such as startup and prime/redundant switchover to confirm implementation is robust.

As the Chandra mission has extended to nearly 15 years, several regions of the payload near the TFTE/bench interface, specifically the Aspect Camera, spacecraft struts, and HRMA struts, all have required relaxation of temperature limits. Although temperatures now drift above planned values, the design conservatism has allowed this happen without serious mission impact. The temperature environment around the HRMA is now approaching the HRMA thermal setpoint, so preliminary discussion of the need to increase the HRMA setpoint is on-going.

\section{Thermal Design and the Error Budgeting Process}

Very early in the Chandra payload design process there was a focus on the thermal contributions to imaging and pointing stability performance. Thermal sensitivities were developed with an Integrated Modeling approach that imposed temperature maps from thermal models into structural models, then with structural deformations integrated into the imaging and line-of-site models. This process led to a good understanding of the thermal impacts on the quality of Chandra science data.

In this process we were able to make hardware design trades favoring the most crucial regions for tight thermal control. For example a HRMA diametrical gradient drives the co-alignment of the entire set of mirror pairs, broadening the PSF, and negatively impacting performance at all $x$-ray energy levels. This resulted in the addition of thermal sensors in a bridge network to explicitly measure gradients across the HRMA. This data has proved valuable in assessing thermal stability of Chandra, and is part of the routine health assessments performed at the Chandra Operations Center.

\section{Thermal Design Lessons from Flight Operation}

Numerous lessons learned have been acquired from the on-orbit operations of the Chandra Observatory through and beyond the 5-year design lifetime. Of those lessons, the majority are associated with the degradation of the thermal control coatings used over the exterior of the Observatory.

While $2^{\text {nd }}$-Surface silvered fused-silica (quartz) mirrors (SSMs) were used for the external radiator surfaces of the Spacecraft (SC) and the Integrated Science Instrument Module (ISIM), these represent far less than $10 \%$ of the total external surface area of the Chandra Observatory. The primary external thermal control coating 
over the SC, Telescope (TS) and ISIM was $2^{\text {nd }}$-Surface Silvered Teflon (SST) also called Flexible Optical Solar Reflector (FOSR), where it was used as a radiator coating in a small number of locations but extensively as the outer layer of multilayer insulation (MLI) blankets - particularly over the sun-side of the Observatory.

When used as the MLI outer layer, the SST material was laminated to an underlying layer of 2-mil Kapton$\mathrm{HN}$ to maintain structural integrity. This laminated construction was selected based on the many years of experience from ground and flight tests (particularly LDEF and SCATHA) as well as correlations of flight data which had determined that the structure of the Teflon layer cracks and erodes over the years of exposure to the flight (charged particle and UV) environments. Prior to launch, all other thermal design features were built, integrated and verified, but no program-specific material degradation tests were performed - the legacy test and flight data provided the basis of the material degradations to be expected during the 5-year design lifetime.

Of the thermal design features verified prior to launch, a key one to note was that virtually all areas of flight $\mathrm{MLI}$ over the SC and TS were verified in test to perform near the extreme low-conductance (low $\mathrm{e}^{*}$ ) range of pre-launch design values - meaning that internal hardware were substantially less sensitive to external thermal effects than required.

Correlations of the SC and TS sub-models of the Observatory Thermal Math Model (TMM) to flight thermal telemetry from both normal-sun and tail-sun attitudes was performed three weeks after launch. These correlations identified four minor modifications needed to SC Propulsion Subsystem (SC-PS) component thermal models to match heater duty-cycles and determined that flight telemetry was best correlated by slight reductions ( $10 \%$ lower $\mathrm{e}^{*}$ than TV-test data correlations) on both the SC and TS external MLI blankets - this effect was expected because of the lower pressure levels at the flight altitudes compared to those achieved in the ground tests of the flight and development hardware.

In early 2002, approximately 30 months after launch the EPHIN sensor temperatures were nearing those predicted for month-60 and an investigation was launched by the factory-support team at TRW Space Park. After extensive evaluation of SC, TS and ISIM temperature telemetry over the full range of Observatory attitudes since launch and re-correlation of the SC and TS TMMs, it was concluded that the SST coating was degrading faster than previously seen in ground tests or on-orbit. The strongest evidence for this came from temperature telemetry on the three assemblies mounted to the sun-side of the Observatory - the Fine Sun Sensors (FSS), the EPHIN instrument and the EPHIN Input Output (EIO) unit, and heater dutycycle data on the TS Optical Bench Assembly (OBA). The correlated degradations were reviewed by NGAS and NASA specialists in the flight charged particle environment who deemed then to be plausible given that the prior design data was from GEO and not the highly-elliptical Chandra orbit - further study was required. Degradation trends from the prior 12-months were extrapolated into the future for the purposes of updating temperature alert/alarm limits on the sun-side components. Even with degradation of the SST coating predicted to significantly exceed the pre-launch year-5 values, all SC components other than those directly mounted to the sun-side panel (EPHIN, EIO and FSS units), were predicted to remain well within their allowable temperature limits through the mission design life - due largely to the extremely lowconductance (low $\mathrm{e}^{*}$ ) performance of the external MLI over the sun-side of the Observatory.

In mid-2005, a study was started to support extension of the Chandra mission life to 15-years. This study evaluated the three years of flight telemetry since the 2002 study including a number of anomalous lowtemp exceedances of SC-PS line limits observed at ranges of Observatory pitch attitudes around 135degrees. The conclusion of this study was that the sun-side components on Chandra continued to degrade at rates similar to those predicted in the 2002 predictions but that only those units mounted directly to the sun-side panel (EPHIN, EIO and FSS units and sun-side SC-PS propellant lines and thruster valves), were in jeopardy of violating hot temperature limits. The anomalous low-temp exceedances of SC-PS line limits at pitch attitudes around 135-degrees was concluded to be the result of the degradation of the SST MLI- 
coating over the line sections - heat transferred absorbed by the degraded $\mathrm{MLI}$ coating transferred through the line-MLI into the line sections where the heater-control thermostats were located, passively maintaining these line locations above thermostat close (turn-on) temperature. The lack of heater power applied to adjacent line sections that are shadowed by the SC-Bus structure at these pitch attitudes (near 135-degrees) cause them to cool below the alert/alarm limit. This issue resulted in an attitude/duration constraint being implemented in the Operations planning CONOPS to avoid this range of attitudes. Long-term degradations were extrapolated from the prior 3-years telemetry data for the purposes of updating alert/alarm limits and additional observation planning.

Correlation of the ISIM TMM sub-model to flight telemetry was also performed by NGAS in late-2005 as well and concluded the following: (1) The ISIM thermal design met all of its 5 year mission life objectives and all component temperatures were within their design limits, (2) The forward section of the ISIM, specifically in the area of the ACIS PSMC and the translation motor, are running hotter than expected as a result of specular solar heating reflections off of the Top Hat second surface mirror radiator and other specular surfaces in that region. The heating in this area was not adequately addressed in the design phase of the program. (3) Updated thermal analyses indicate that most ISIM components will remain within their design limits at the 15 year life extension goal, with the exception of, ACIS PSMC, translation motor, ACIS focal plane, the translation table at the interface with the ACIS attachment location and the HRC CEA, Detectors, and detector housing for $\mathrm{HRC}$ in viewing position.

Mission constraints have been identified that allow the PSMC to be maintained within its currently defined yellow/red limits. These constraints include limiting the allowable sun angles, and/or reducing the operating configuration of ACIS. The reduced operating configuration can be attained by reducing the number of operating CCDs, or in the case when HRC is in view by going to the ACIS standby mode.

Since 2006, temperatures have continued to slowly trend toward higher levels over the observatory without other notable thermal anomalies or issues. Thermal support have been provided as needed for anomalies on SC-PS components to support life-extension studies of individual components or subassemblies.

The lessons-learned from nearly fifteen (15) years of support to the on-orbit operations of the Chandra observatory are:

1) On-orbit degradation of the SST coating has been substantially greater than predicted prior to launch.

a. Selection of the SST coating was not required for the SC Bus - alternative coatings, particularly include black Kapton would have met requirements over the entire SC Bus and PS components with far less on-orbit degradation and would have avoided the current attitude constraint sources necessitated by the Bus temperature exceedances.

i. Note such a material change would have had to be implemented shortly after the Thermal Control Subsystem (TCS) PDA in August 1994 to have been successful as 4-5 propellant line heater circuit layouts would have been substantially different given the change in coating of their external MLI surfaces.

b. Significantly more extensive use of quarts SSMs instead of SST tape and MLI coatings should have been implemented to maintain cold-biasing of units on the sun-side of the SC-Bus (EPHIN, EIO and FSS units).

c. Alternative $\mathrm{MLI} / \mathrm{SLI}$ shielding concepts could have been implemented had the true degradation been known or anticipated.

d. The correlated degradation of the SST coating is now so extreme that a logical conclusion is that its emittance has actually begun to be reduced due to material thickness reductions/erosion. 
2) Temperature telemetry on external PS propellant lines should have been more extensive - the quantity of flight sensors provides very valuable indication of the most sensitive component responses to short-term changes (attitudes/seasons) as well as long-term changes (coating degradations).

\section{Calibration}

The Chandra telescope, instruments, and the calibration facility are all complex sub-systems that were not completely understood until calibration commenced. The challenging nature of calibration became apparent to the test team after the results of the VETA-I test were analyzed. Chief among these concerns was the low efficiency of data collection, defined as the quotient of the time collecting $x$-ray data divided by the total clock or calendar time. For the VETA-I test this efficiency was about 1-3\%. It was clear that improvements needed to be realized to achieve the flight calibration goals. To this end, a series of rehearsal tests, were conducted, spanning approximately May 1996 to late November 1996 (when the HRMA arrived at MSFC). These tests were carefully designed to see where time was being spent in the measurement process and to the extent possible, reduce that overhead, improve instrument and facility operations and communications between project science and the test conductors responsible for producing the test procedures. When it was time for the flight calibration, we had in place systems that could produce an entire new test procedure from the science database of requested measurements in 30 minutes. This gave us great flexibility in test execution. Finally, the rehearsals, did prove effective, the final average efficiency for x-ray calibration was $48.8 \%$ for all test phases. The efficiency for the non-flight detector was significantly lower than for calibration with the flight detectors. A good lesson here is that rehearsals pay off, but we would have even better served with a "ground" mode allowing more rapid data collection.

The calibration plan, although it had been thought out and planned in detail, needed considerable revision and updating during the calibration in response to the data being collected. To make sure that the calibration team had this flexibility, each shift (we were operating $24 / 7$ at this point) had a senior project scientist who could red-line the test procedure Following each morning's 7 am shift change meeting, the calibration (re)scheduling meeting made needed changes to the calibration database to accommodate hardware issues or additional required measurements that had been identified. This revised database was handed to the TRW test conductor, and the new procedure was implemented on the " $\mathrm{B}$ " shift. All of this required close coordination and knowledge among all those involved in calibration. This revision required some calibration team members to come in early and support additional meetings, the test team rose to the challenge.

\section{Vacuum Ground Support Equipment}

Due to limited resources and great efforts to stay on the critical test and delivery schedule, the ground support equipment for use at XRCF did not get a full check out prior to use. Namely, it was never put into the vacuum chamber and tested as a system. The HRMA OGSE was designed well and checked out in Rochester and appeared to be working as designed but vacuum testing after integration of the flight hardware identified many issues that were not detected prior to vacuum testing. Early vacuum testing would have identified problems and corrected them off the critical path of the program, which ran right through x-ray calibration. We got it all working, but earlier system debugging would have identified these issues and they could have been solved off the critical path

\section{Testing}

Before launch of any remotely operated satellite or other equipment, perform end- to- end tests of the flight system with the ground control system using the final flight and ground versions of hardware, 
software, database and procedures. The flight operations team should perform these tests. To the extent possible, each command type should be sent and verified. Commands that by their nature cannot be tested in this manor should be tested in a high fidelity simulator with the ground system and all hardware commands to the flight system should be verified using the ground system for functionality.

Taking shortcuts in box level testing is a gamble with bad odds. Spend the time to thoroughly verify the flight worthiness of hardware and software at the lowest level. Finding box level problems during systems level testing is very costly and disruptive to schedule.

If independent review teams are to be used in a program, then get them in at PDR and keep them involved in all major reviews as well as in the resolution of critical issues as they arise throughout the program.

\section{ACIS radiation damage}

It was known before launch that protons with energies around $100 \mathrm{keV}$ could damage the CCDs. It was NOT known that $\sim 100 \mathrm{keV}$ protons could scatter off of the surfaces of the mirrors in the HRMA and be crudely focussed toward the focal plane. The ACIS design would have benefited from having a filter with multiple positions with one of the positions blocking light and low energy particles, such as a thin Al sheet. The framestore section of the ACIS CCDs is covered by $1 / 8$ inch of gold-plated Al. There has been no measurable damage in the framestore regions of the CCDs, so we know that $1 / 8$ inch of $A$ is sufficient to stop most of the low energy protons. Alternative to a filter with multiple positions, ACIS could have been designed to have a highly reliable door that was designed to be opened and closed many times.

We unintentionally exposed ACIS to low energy protons for 7 radiation belt passages early in the mission because we did not want to exercise the translation mechanism on the Science Instrument Module (SIM) until ACIS had collected some data on celestial sources. The external calibration source for ACIS is only visible when the SIM has translated to put ACIS in the stow position. Once we had translated the SIM and collected data from the ECS, it was obvious that the spectral resolution of the CCDs had degraded due to the radiation damage. We should have had a means of monitoring the ACIS performance sooner than after 7 orbits. We should have been monitoring the ACIS performance as soon as the door was opened and ACIS was exposed to radiation coming from the direction of the HRMA. We could have done this by translating the SIM sooner to get under the ECS or we could have designed a calibration source that could be used when ACIS is in the focal position. We have now translated the SIM literally thousands of times in the mission and the mechanism has proven to be extremely reliable. They key is you must have a standard set of tests that can rapidly detect changes in the instrument performance and you must execute those tests frequently at the beginning of the mission.

\section{Contamination}

The ACIS optical blocking filter (OBF) is the coldest surface that faces the interior of the Optical Bench Assembly(OBA) on Chandra. The edges of the filter were at $-60 \mathrm{C}$ and the center was at -50/-45 C (ACISS/ACIS-I) from launch until April 2008. In April 2008, we turned off the ACIS DH heater so that the edges of the filter fluctuate between $-72 \mathrm{C}$ and $-60 \mathrm{C}$. The ACIS CCDs are much colder at-120 C but in a mostly sealed volume behind the filter. A significant contamination layer has built up on the ACIS OBF and continues to accumulate at this point in the mission. Since the OBF is the coldest surface that views the OBA volume, any particle that is liberated inside the volume could potentially make its way to the OBF and freeze out on that surface. The plan before launch was to bake ACIS out regularly. A bakeout early in the mission exacerbated the radiation damage by increasing the mobility of the radiation traps when the temperature of the CCDs was raised to room temperature. Therefore, no bakeouts to room temperature have been attempted with ACIS to possibly remove the contamination on the filter. 
The ACIS design would have been better if there was a surface colder than the OBF that would act as a getter for the contaminant. Second, ACIS has only two heaters, one on the focal plane and one on the Detector Housing. It would have been better if ACIS had had a heater that would heat only the filter. Third, the only efficient vent for contaminants to exit the spacecraft is out through the HRMA on the opposite of the OBA from ACIS. There should have been an effective vent closer to ACIS. Fourth, the graphite-expoxy structure of the OBA is unshieled on its interior. Other satellites have a layer of MLI or Al foil that lines the inside of the optical cavity to act as a contamination barrier.

\section{X-ray Calibration Facility (XRCF)}

One of the key 'take aways' from the Chandra program was the early involvement and efforts on the XRCF. MSFC was rebuilding the XRCF for the main purpose of testing the HRMA in its most flight like configuration. The MSFC team along with the TRW/Kodak and supporting facility teams engaged in many interface control and specification development technical exchange meetings. These meetings were conducted prior and during the facility rebuild. The early on collaboration was paramount in developing the interfaces and the specifications of the facility, test equipment and special equipment. The early on development of these key requirements laid the foundation for the sound design of all of the equipment necessary for a successful verification and calibration test program.

As the MSFC test facility was in its final stages the assembly and test teams were actively involved and engaged in facility training and certifications for supporting team members at MSFC. This may sound trivial but a key success was having qualified test team members with the proper training and authority to perform tasks within the facility. These afforded opportunities to not only remain on schedule but to improve schedule and more importantly provide alternatives when unforeseen events occurred.

The rehearsal activities discussed about were aslo key to the successful operation activation of the test facility. As we were constructing the facility, we took the time to plan and execute as many incremental, subsystem-level performance verification test activities as possible. This prevented us from finding problems during the full system-level activation that would have had the potential to impact the schedule significantly. The lesson here is that we planned to perform these activation tests and executed that plan many times, sub-system verification activities such as these get deleted or deferred in order to save cost/schedule. The result was that the VETA and HRMA testing were completed w/o any test facility-related interruptions.

\section{Summary and a Final Word}

The paper presented above has given some detailed lessons learned for $\mathrm{x}$-ray telescope design and implementation. $[7,9,10]$ In trying to summarize our experience five major themes arise, they are presented in no particular order;

1. Build a solid team - face to face, keep that team together throughout the program.

2. Understand the details---there is no such thing as a "simple" interface or material property.

3. Understand the risks and attack the big ones as early as possible.

4. Practice all challenging or new processes, procedures, facilities and teams off the critical path

5. Test completely as early and completely as possible, after all "An ounce of prevention is worth a pound of cure." 
In the end, the Chandra team worked to a single goal, that of building and flying an instrument to provide mankind a new view on the x-ray universe. It was hard, it was exciting, it was worthwhile and ultimately successful. The Chandra team wishes the same success it has achieved to the teams that work the missions to come and hope that these lessons can contribute to their successes.

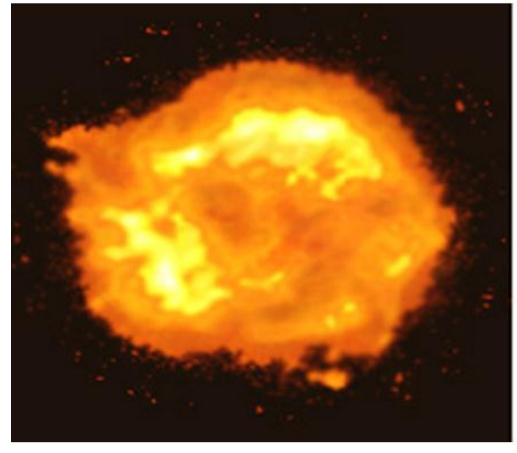

Rosat Image

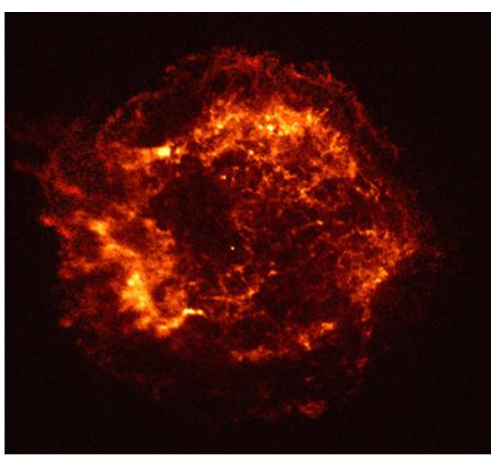

Chandra Image

Figure 8: Improved Imagery from Chandra

\section{References}

[1] M. C. Weisskopf, "Fifteen years of Chandra operation: scientific highlights and lessons learned", SPIE Paper 9144-24, this conference.

[2] D. A. Schwartz, " Development lessons from the AXAF/Chandra X-ray observatory" Paper 9144-27, this conference.

[3] Martin C. Weisskopf; AXAF VETA test: an overview. Proc. SPIE 1742, Multilayer and Grazing Incidence XRay/EUV Optics for Astronomy and Projection Lithography, 2(January 21, 1993); doi:10.1117/12.140547.

[4] William A. Podgorski ; Kathryn A. Flanagan ; Mark D. Freeman ; Richard E. Goddard ; Edwin M. Kellogg ; Timothy J. Norton ; J. P. Ouellette ; Adrian G. Roy ; Daniel A. Schwartz; VETA-I x-ray detection system. Proc. SPIE 1742, Multilayer and Grazing Incidence X-Ray/EUV Optics for Astronomy and Projection Lithography, 40 (January 21, 1993); doi:10.1117/12.140590.

[5] Jonathan W. Arenberg ; Scott C. Texter; Motion detection system for AXAF x-ray ground testing. Proc. SPIE 1742, Multilayer and Grazing Incidence X-Ray/EUV Optics for Astronomy and Projection Lithography, 104 (January 21, 1993); doi:10.1117/12.140594.

[4] Leon McKendrick, Daniel Shropshire, "Chandra Operations Lessons Learned", Space 2004 Conference and Exhibit, Paper 6040, American Institute of Aeronautics and Astronautics, 2004, doi:10.2514/6.20046040

[5] Keith Hefner, Greg Davidson, "Performance as Promised: The Chandra X-ray Observatory", Space 2004 Conference and Exhibit, Paper 5935, American Institute of Aeronautics and Astronautics, 2004, doi:10.2514/6.2004-5935

[6] Steven Jordan and Scott Texter," The Science Instrument Module for the AXAF mission", Space Programs and Technologies Conference and Exhibit, American Institute of Aeronautics and Astronautics, 1994, doi:10.2514/6.1994-4565 
[7] Gary Matthews ; Keith Havey, Jr.; Ten years of Chandra: reflecting back on engineering lessons learned during the design, fabrication, integration, test, and verification of NASA's great x-ray observatory. Proc. SPIE 7738, Modeling, Systems Engineering, and Project Management for Astronomy IV, 77380Y (August 03, 2010); doi:10.1117/12.858268

[8] S. Texter, J. Arenberg, R. Carlson, G. Grimm, "HRMA X-ray Test and Calibration, SI Calibration Report", XC04, Delivered to MSFC in fulfillment of contract NAS8-37710

[9] Jean Oliver, "Chandra X-ray Observatory (CXO) Development Program Programmatic "Lessons Learned"", URL: http://lis.nasa.gov/lesson/0987, last accessed 24 May 2014.

[10] Tony Lavoie, "Chandra Lessons Learned", URL http://ocw.mit.edu/courses/aeronautics-andastronautics/16-885j-aircraft-systems-engineering-fall-2005/video-lectures/lav_chan_sys_eng.pdf, last accessed 24 May 2014. 